\title{
Mathematical treatment of chemiluminescence data allowing an optimised kinetic analysis of vascular NAD(P)H-dependent superoxide anion production
}

\author{
J.-P. Iliou*, N. Villeneuve, M.-P. Fournet-Bourguignon, F. Robin, C. Jacquemin, V. Lestriez, C. Petit,
} A. Pillon and J.-P. Vilaine

Institut de Recherches Servier, Division Pathologies Cardiaques et Vasculaires, 11, rue des Moulineaux, 92150 Suresnes, France

* Corresponding author: jpiliou@ netgrs.com

Regulation of vascular redox homeostasis plays a central role in the control of vascular tone (e.g.: redox modulation of endothelial NO synthase and soluble guanylate cyclase activities). Superoxide anion is one of the main reactive oxygen species involved in the modulation of the redox status of the vessel. It is crucial to know if pathological situations or drug treatments are able to modify NAD(P)H oxidase-dependent production of $\mathrm{O}_{2}^{-}$. As a steady-state approach is the only way for a valid assessment of these parameters, a mathematical treatment allowing visualisation of the linear portion of the initial velocity of $\mathrm{O}_{2}^{-}$production was developed. Using two vascular preparations (rat aortic rings and mouse thoracic aortae), the chemiluminescence $(C L)$ in the presence of lucigenin was recorded every second in the 15-30 minute period after successive injections of NADPH and superoxide dismutase (SOD). Because, both $\mathrm{O}_{2}^{-}$and photons are labile and evanescent products, visualisation of the steady-state requires calculation of their cumulative production by integral calculus using a first order integral equation. The cumulative production of $\mathrm{CL}$ and/or $\mathrm{O}_{2}^{-}$was plotted as a function of the recording time. The initial velocities, as a function of the NADPH concentrations, were determined from the linear portion of these plots. For a precise molar quantification of $\mathrm{O}_{2}^{-}$ production, a calibration curve of initial velocities using xanthine oxidase + xanthine as a source of $\mathrm{O}_{2}^{-}$was plotted by comparing $\mathrm{CL}$ and cytochrome $\mathrm{C}$ reduction. The NADPH-dependent CL production exhibited Michaelian behavior and SOD acted as a noncompetitive inhibitor. For example, the values for NADPH oxidase in rat aorta were: $\mathrm{Km}=237 \pm 2 \mu \mathrm{M}$; Vmax $=$ 500-600 pmol $\mathrm{O}_{2}-/ \mathrm{min} /$ ring.

\section{Introduction}

The superoxide anion is one of the most important physiopathological modulators of stationary and efficient NO concentration in blood vessels [1] and figure 1 shows a general view of its vascular pathways. Indeed, the product of the covalent interaction between $\mathrm{NO}$ and $\mathrm{O}_{2}^{-}, \mathrm{ONOO}^{-}$, does not exhibit relaxant properties comparable to those of $\mathrm{NO}$ [2]. The main vascular source of $\mathrm{O}_{2}^{-}$is the membrane $\mathrm{NAD}(\mathrm{P}) \mathrm{H}$ oxidase. This enzymatic activity has been detected in each vascular tissue type: endothelium, smooth muscle and adventitia [3-5]. Reductase moiety of endothelial NO synthase (eNOS) is also able to generate $\mathrm{O}_{2}^{-}$using similar reaction with NADPH consumption [6,7]. The main metabolic pathway of $\mathrm{O}_{2}^{-}$in the absence of NO is the dismutation into $\mathrm{H}_{2} \mathrm{O}_{2}$ and $\mathrm{O}_{2}$ in the presence of protons. The dismutation is a spontaneous process amplified by superoxide dismutase (SOD) activities [8].

For a better knowledge of the mechanisms involved in modification of redox homeostasis associated with numerous vascular pathologies, it is very important to evaluate both the activity of $\mathrm{NAD}(\mathrm{P}) \mathrm{H}$ oxidase and the related $\mathrm{O}_{2}^{-}$production. For a complete description of NAD(P)H-dependent $\mathrm{O}_{2}^{-}$production in vessel in function of the physiopathological status or pharmacological treatment, it is necessary to obtain consistent and precise information about enzymatic parameters such as Vmax and $\mathrm{Km}$.

One of the most used techniques for $\mathrm{O}_{2}^{-}$detection in vascular preparation is chemiluminescence (CL): measurement of the light produced by the interaction between $\mathrm{O}_{2}^{-}$and a $\mathrm{CL}$ probe. The main used $\mathrm{CL}$ probe for $\mathrm{O}_{2}^{-}$in the literature is lucigenin $\left(\mathrm{Luc}^{2+}\right)$; there are also few papers using imidazopyrazinone derivatives $[9,10]$. The CL is an indirect technique for $\mathrm{O}_{2}^{-}$detection, there are also restrictive conditions of interpretation when $\mathrm{Luc}^{2+}$ is used as probe [10-12].

The present paper describes a mathematical treatment of CL data which allowed to determine $\mathrm{Km}$ and Vmax of $\mathrm{NAD}(\mathrm{P}) \mathrm{H}$-dependent $\mathrm{O}_{2}^{-}$production in vascular preparations. The experimental evaluation of these parameters 


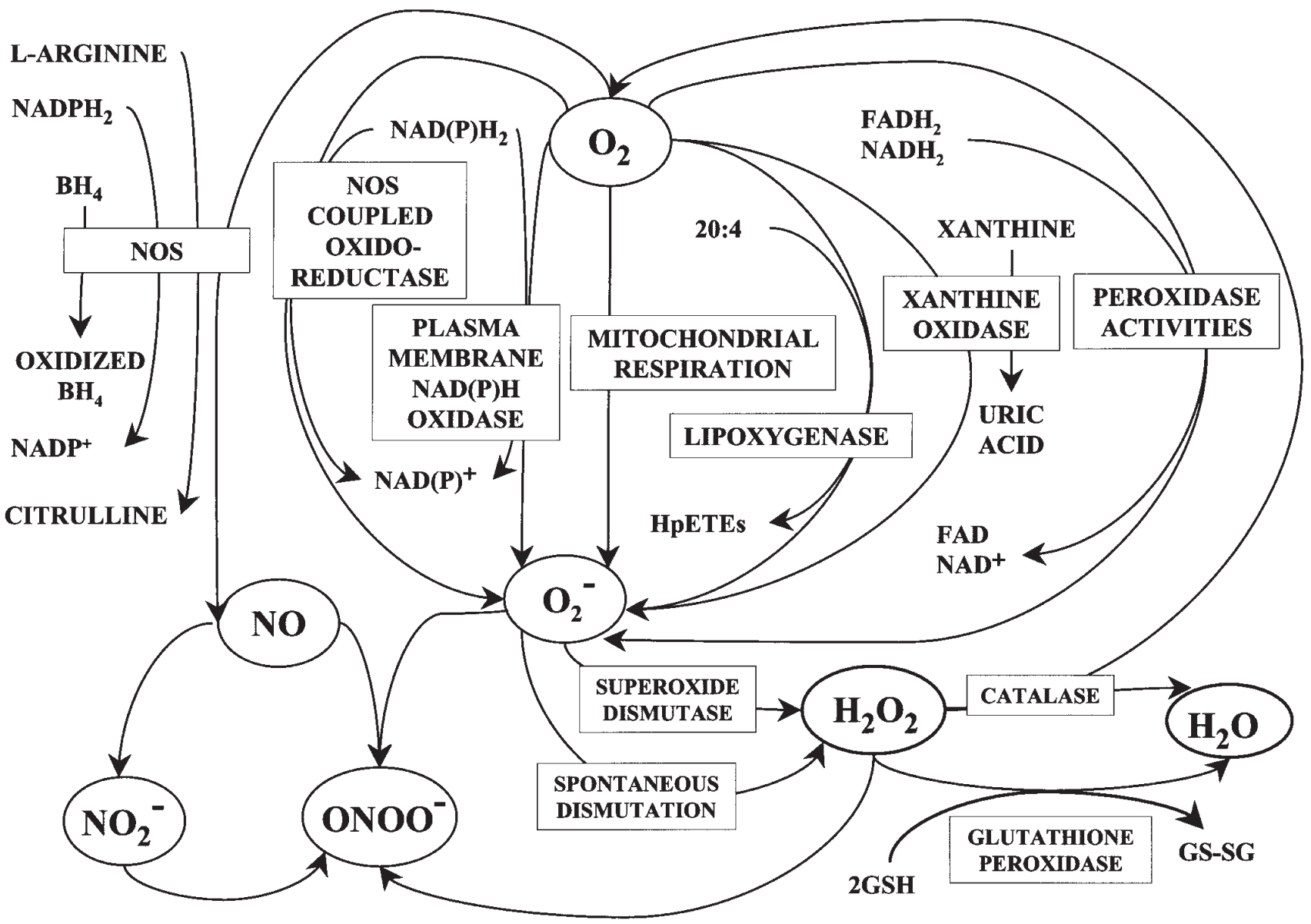

Figure 1. General description of vascular pathways of $\mathrm{O}_{2}^{-}$. The equation of in vitro synthesis production of $\mathrm{ONOO}^{-}$by covalent reaction between $\mathrm{NO}_{2}^{-}$and $\mathrm{H}_{2} \mathrm{O}_{2}$ in acidic condition [25] has been added in the scheme indicating that direct metabolites of $\mathrm{NO}^{-}$and $\mathrm{O}_{2}^{-}$would be considered as potential precursors for ONOO- generation. BH4: tetrahydrobiopterin; GSH: glutathione; GS-SG: oxidative derivative of glutathione; HpETEs: hydroperoxyeicosatetraenoic acids or hydroperoxidized derivatives of arachidonic acid (20:4); NOS: nitric oxide synthase.

involves respect of the classic steady-state conditions [13]. Figure 2 summarises briefly the steady-state conditions characterised by constant rates of substrate consumption and product generation (initial velocities). During this period, the instantaneous rate of transformation of the substrate into product is constant. The slope of product generation, corresponding to initial velocity, is calculated from the cumulative product data. Because both $\mathrm{O}_{2}{ }^{-}$and photons are labile and evanescent products, the experimental CL recording (Fig. 3), representative of the instantaneous formation, did not give direct information about their cumulative production. The use of first order equation of integral calculus avoids this obstacle. The present paper illustrates the principle of this data treatment using two vascular preparations: rat aortic rings and mouse aortae. The physiopathological interest of the data treatment method is also indicated by the comparison of aortae from hypercholesterolemic mice versus aortae from control mice.

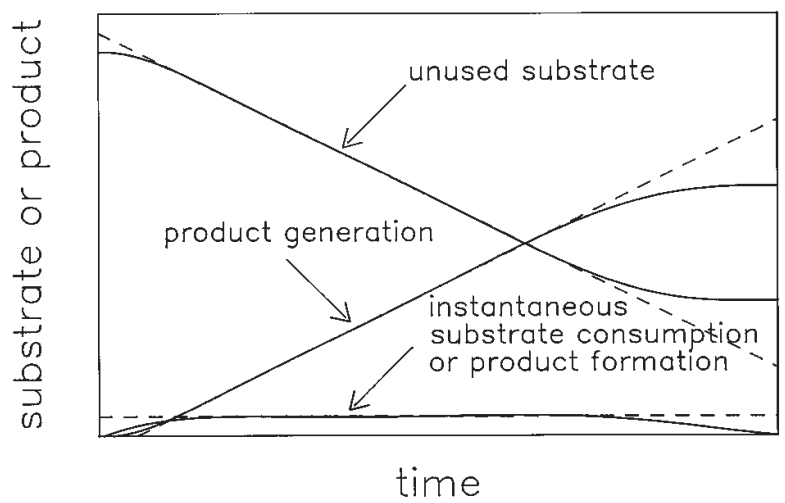

Figure 2. Steady-state conditions and initial velocity. Scale proportions between the 3 traces are strictly respected. Because $\mathrm{O}_{2}^{-}$ and photons are obviously evanescent products, the plateau of the direct recorded CL signal is only representative of the rate of instantaneous product formation (see figure 3). A mathematical treatment of these data is necessary to obtain information about the rate of cumulative product generation. 


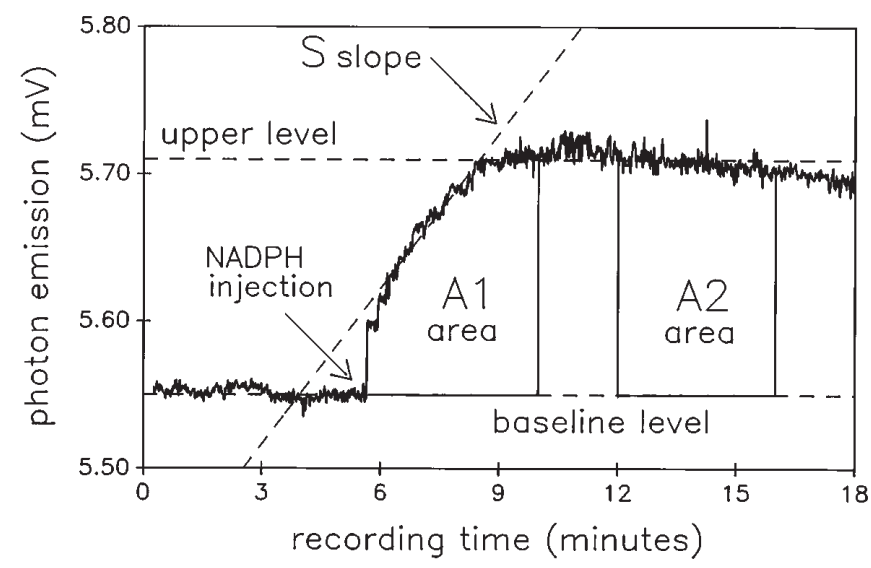

Figure 3. Representative recording of $\mathrm{CL}$ signal induced by injection of $100 \mu \mathrm{M}$ NADPH to one rat aortic ring in the presence of $250 \mu \mathrm{M}$ Luc $^{2+}$. Sampling rate: one measurement per second. Vertical distance between upperline and baseline: maximum amplitude signal. Slope S: variation of instantaneous rate during initiation phase. Zone A1 or A2: area under the curve for a standardized time of recording.

\section{Materials and methods}

\section{Chemicals}

Cytochrome C (oxidised form) from horse heart, SOD from bovine erythrocytes, xanthine oxidase from buttermilk, $\mathrm{Luc}^{2+}$, NADPH (preweighted vials) and xanthine were obtained from Sigma (Saint Louis, USA). NO gas was purchased from Praxair (Oevel, Belgium). All others chemicals were of analytical grade and obtained from Merck (Darmstadt, Germany).

\section{Preparation of electrochemically calibrated aqueous solution of NO}

Calibrated solution of NO in oxygen-free distilled water was prepared extemporaneously as indicated [14]. The concentration of $1.8 \mathrm{mM} \mathrm{NO}$ solution is stable during 20 minutes at $4{ }^{\circ} \mathrm{C}$.

\section{Animal ethics}

All procedures were carried out in accordance with the guidelines of the French Ministry of Agriculture for the use and care of laboratory animals.

\section{Vessel preparations}

Male Wistar rats (425-450 g) were purchased from Charles Rivers Laboratories (Saint-Aubin-les-Elbœuf, France), C57BL/6J mice from Iffa-Credo (L'Arbresle, France) and apolipoprotein E-deficient (ApoE KO) mice from Transgenic Alliance (L'Arbresle, France). Animals were anaesthetised with sodium pentobarbital ( $30 \mathrm{mg} / \mathrm{kg}$ ip). The thoracic aorta was carefully removed and immediately placed in modified Krebs bicarbonate solution containing (in $\mathrm{mM}$ ): $\mathrm{NaCl}$ (120.3); $\mathrm{KCl}$ (4.8); $\mathrm{CaCl}_{2}$ (2.5); $\mathrm{KH}_{2} \mathrm{PO}_{4}$ (1.2); $\mathrm{MgSO}_{4}$ (1.3); $\mathrm{NaHCO}_{3}$ (24.2); glucose (11.1) and bubbled with a mixture of $\mathrm{O}_{2} / \mathrm{CO}_{2}(95 \mathrm{vol}: 5 \mathrm{vol})$ at $\mathrm{pH}$ 7.4. Aortae were cleaned of connective tissues. The total thoracic aortae from mice, including the arch, were used. In rat, 4 rings $(3-4 \mathrm{~mm}$ length) were cut from the aortic arch through the thoracic aorta. Rat aortic rings were then allowed to equilibrate for 30-150 minutes at $4{ }^{\circ} \mathrm{C}$ before initiating the experiment. Mouse aortae were equilibrated 20-30 minutes at room temperature before CL recording.

\section{CL procedure}

Experiments were performed using the luminometer BioOrbit 1251 (Labsystems, Helsinki, Finland) because this instrument allowed (1) manual dispensing of reagent directly into the measuring chamber and (2) continuous mixing of the sample by alternative rotation of the cuvette in the measuring chamber. This last point is crucial for a homogenous and a reproducible repartition of the injected reagents into the sample cuvette. After equilibrium period, sample cuvette containing one rat aortic ring or one mouse aorta in $1 \mathrm{~mL}$ Hepes/Tris buffer (pH 7.4) supplemented with $250 \mu \mathrm{M} \mathrm{Luc}{ }^{2+}$ was prewarmed $5 \mathrm{~min}$ at $37{ }^{\circ} \mathrm{C}$ into the luminometer. The global composition of Hepes/Tris buffer (20 mM Hepes adjusted at $\mathrm{pH} 7.4$ with added Tris) without glucose was (in $\mathrm{mM}$ ): $\mathrm{NaCl}$ (140); $\mathrm{KCl}$ (5.4); $\mathrm{CaCl}_{2}$ (2.4) and $\mathrm{MgSO}_{4}$ (0.7). The baseline CL signal was then recorded during 5 minutes before injection of NADPH. The signal acquisition and treatment procedures were detailed hereafter.

\section{CL data acquisition}

For preliminary and validation experiments, the electrical CL signal generated by the luminometer was digitalised using a sampling rate of one measurement per second and exported to a chromatographic software (Millennium, Waters S.A., Guyancourt, France) able to transform the raw data in a format adapted to a conventional spread sheet software for calculus treatment. Now, a specific software able to do both the screen plot of the recording and the calculus from raw data generated by the luminometer has been edited (further information available from the authors).

\section{Cytochrome $C$ reduction procedure}

For a precise molar quantification of $\mathrm{O}_{2}^{-}$production by $\mathrm{CL}$ measurement, a calibration curve using xanthine oxidase + xanthine as a source of $\mathrm{O}_{2}^{-}$was plotted by comparing $\mathrm{CL}$ with cytochrome $\mathrm{C}$ reduction. Reduction of $25 \mu \mathrm{M}$ cytochrome $\mathrm{C}$ was performed at $549 \mathrm{~nm}$ and at $37{ }^{\circ} \mathrm{C}$ using a Kontron-Uvikon 860 spectrophotometer (Zurich, Switzerland). The initial velocity corresponded to the slope of the linear portion of the recording. The same work solutions of xanthine oxidase (final concentrations from $100 \mu \mathrm{U} / \mathrm{mL}$ to $2 \mathrm{mU} / \mathrm{mL}$ ) and xanthine (final concentration $30 \mu \mathrm{M}$ ) were tested both for CL production and cytochrome 
$\mathrm{C}$ reduction. A molar extinction coefficient of $21000 \mathrm{~cm}^{-1}$ at $259 \mathrm{~nm}$ was used for quantification of reduced cytochrome $\mathrm{C}$.

\section{Results and discussion}

\section{Principle of the mathematical treatment of CL data}

Figure 3 shows a representative recording of the light production induced by the addition of NADPH to rat aortic ring in the presence of $\mathrm{Luc}^{2+}$. Three methods are classically described in the literature to quantify this measure. The first method, the simplest, is the measurement of the maximum

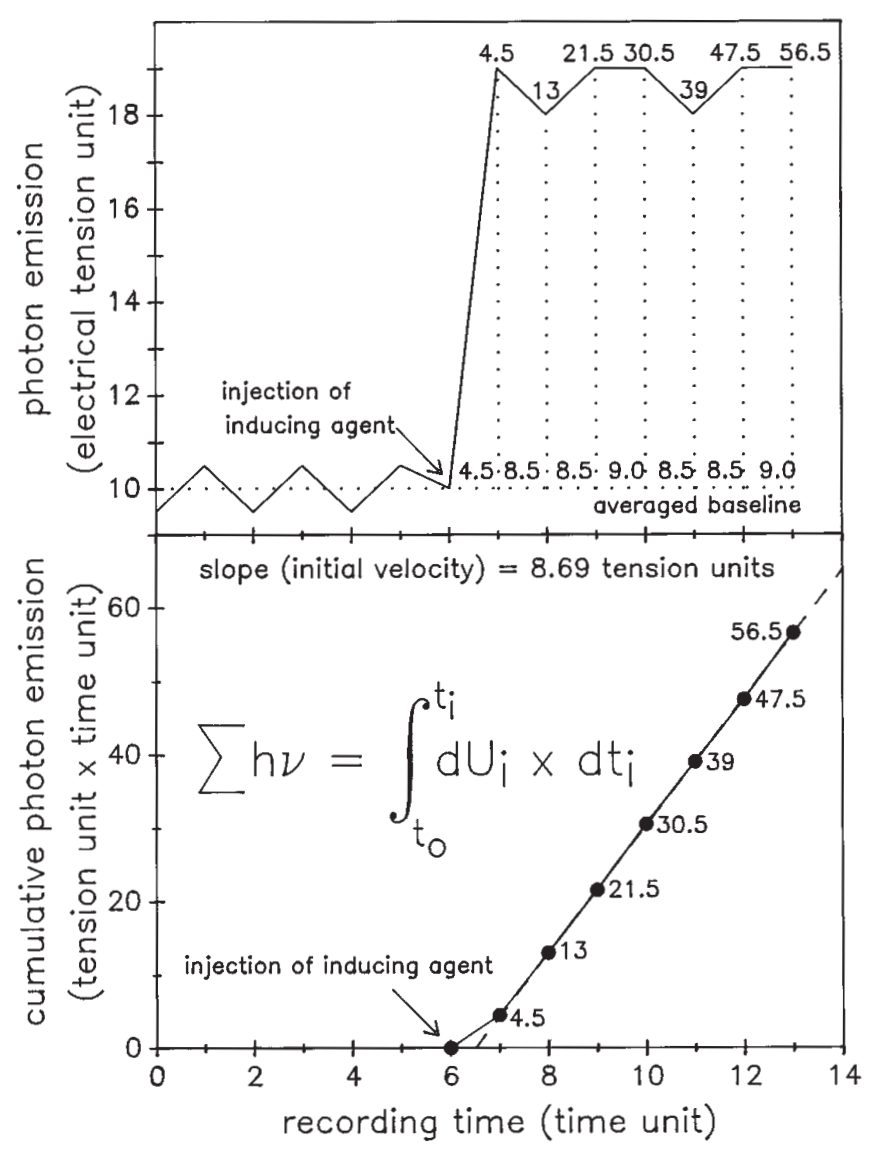

Figure 4. General principle for determination of initial velocity of $\mathrm{O}_{2}^{-} /$photon production. The upper panel is extrapolated from figure 3 . The photon production between two successive sampling points is equivalent to the area of corresponding triangle or trapeze. Each successive area value is indicated above averaged baseline. Cumulative area values after each sampling point are indicated at the top of recording. The lower panel plots the cumulative area values in function of recording duration as indicated by the integral equation. The slope of the linear phase is equivalent to the initial velocity and corresponds to the plateau phase of instantaneous rate (upper panel). amplitude of the signal (the vertical distance between baseline and upperline); numerous authors used this method [1517]. The second method is the measurement of the slope (S segment of figure 3) of the initial light production [3]. The third method $[12,18]$ measures the area under the curve for a standardised time of recording (zone A1 or A2). These 3 methods are not able to give consistent information about Michaelis-Menten parameters of vascular NAD $(\mathrm{P}) \mathrm{H}$ oxidase activity. The maximum amplitude (distance between baseline and upperline) doesn't give information about the existence and/or duration of the initial velocity conditions. The slope $\mathrm{S}$ gives information about the increasing of instantaneous velocity of CL emission during initiation phase of enzymatic catalysis but not about the initial velocity of $\mathrm{O}_{2}^{-}$production during steady-state conditions. The areas A1 and A2 characterise only the total photon production during a specified period but do not indicate if steady state conditions are respected. The figure 4 describes the general method for mathematical treatment of CL data allowing determination of the steady-state condition. The upper panel of figure 4, extrapolated from figure 3 , shows a fraction of the recording around the NADPH addition. The sampling rate is one measurement per period. The light produced during the first period following the NADPH addition corresponds to the triangle area (value: 4.5 tension unit $\times$ period unit). The light produced during the second period of sampling after NADPH addition corresponds to the area of the trapeze at the right side of the triangle (value: 8.5 tension unit $\times$ period unit). For example, during the first 3 periods after NADPH addition, the cumulative $\mathrm{CL}$ production corresponds to 21.5 area units $(4.5+8.5+8.5=21.5)$. On the lower panel of figure 4 , the cumulative photon production is plotted as a function of the recording time using the indicated first order integral equation. The initial velocity of photon production is determined from the linear portion of the plot. If $\mathrm{CL}$ signal is expressed as $\mathrm{mV}$ and time duration as min, the cumulative photon emission is expressed as $\mathrm{mV} \times \min$ and the initial velocity as $\mathrm{mV}$ (for a specified volume of incubating sample).

\section{Visualisation of steady state condition for NADPH oxidase activity in rat aortic rings}

Figure 5 is the experimental illustration of the mathematical principle exposed above. When NADPH $(100 \mu \mathrm{M})$ is injected on rat aortic ring in the presence of $\mathrm{Luc}^{2+}$ (Fig. 5, upper panel), a photon emission rised rapidly to a plateau was observed. After plateau stabilization, SOD (300 U/mL) was injected. The photon emission rate decreased by $44 \%$ in less than 2 min and rapidly a new plateau was observed. The cumulative photon emission, calculated as indicated figure 4 , is plotted on the lower panel of the figure 5. Two linear phases were obtained corresponding to the plateau phases of the original recording (upper panel). The difference between the slope values $(0.237-0.126=0.111 \mathrm{mV})$ is indicative of the exogenous formation of $\mathrm{O}_{2}^{-}$accessible to added SOD. Injected SOD is not able to penetrate into vascular cells and to degrade intracellular $\mathrm{O}_{2}^{-}$. In the absence of aortic rings, only a transient injection artifact was 


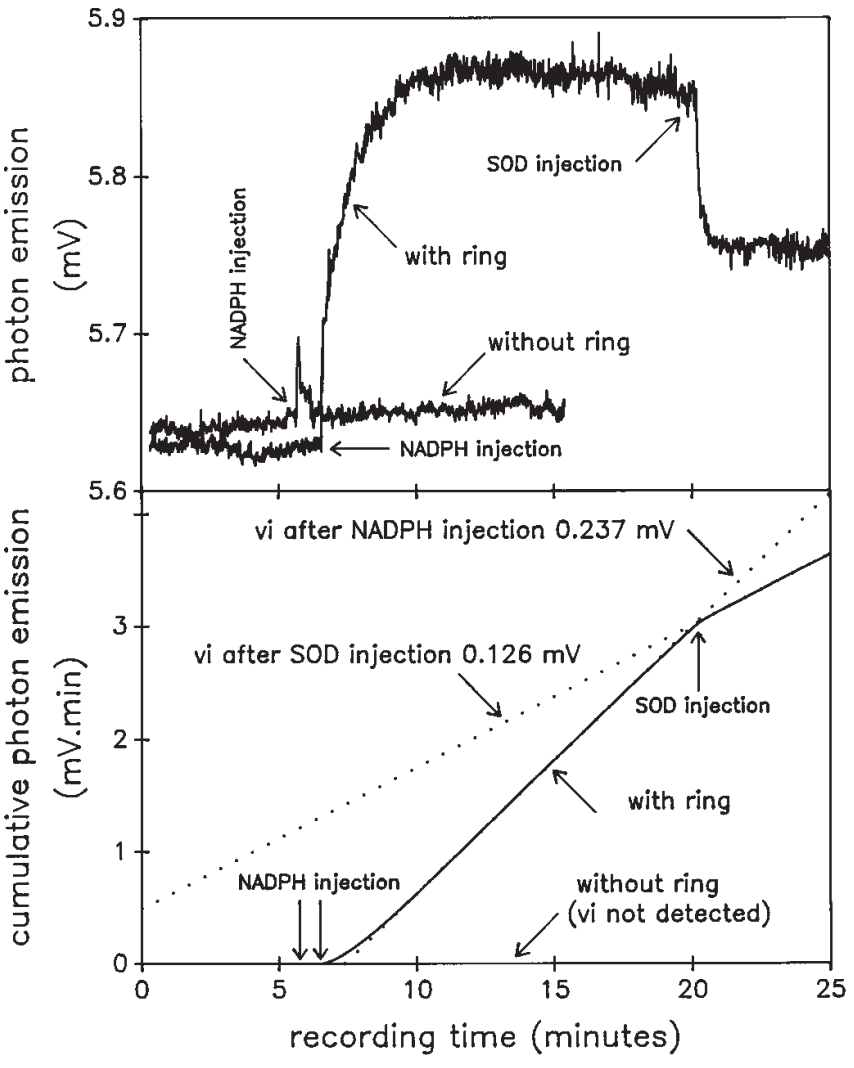

Figure 5. Visualization of initial velocity for NADPH oxidase activity in rat aortic ring. Upper panel: experimental recording of $\mathrm{CL}$ production induced and modulated by successive injections of $100 \mu \mathrm{M}$ NADPH and $300 \mathrm{U} / \mathrm{mL}$ SOD in a cuvette containing one rat aortic ring preincubated with $250 \mu \mathrm{M} \mathrm{Luc}{ }^{2+}$ at $37{ }^{\circ} \mathrm{C}$. Final volume in the cuvette: $1000 \mu \mathrm{L}$. Record of $\mathrm{CL}$ emitted by a control cuvette without ring is also plotted. Lower panel: initial velocities (vi) of photon production are calculated as indicated in figure 4 . In the absence of vascular preparation, cumulative photon production is not detected. The high sampling rate (one measurement per second) gives directly linear profiles that do not require smoothing. Units are $\mathrm{mV}$.min and $\mathrm{mV}$ for cumulative photon production and initial velocity, respectively.

observed after addition of NADPH without significant photon emission. The high sampling rate $(1$ measurement per second) allows to obtain directly linear profiles that do not require smoothing. The slope were determined using linear regression analysis.

If $14 \mathrm{nmol}$ of NO (electrochemically calibrated aqueous solution) was injected during the initial velocity phase observed after NADPH addition, a complete but transient extinction of the photon emission was observed (Fig. 6). After 20-40 seconds of transient extinction, corresponding to the complete consumption of added $\mathrm{NO}$ by the continuous source of $\mathrm{O}_{2}^{-}$, photon emission restarted following steadystate condition but at a lower level. If during this second plateau, SOD $(300 \mathrm{U} / \mathrm{mL})$ was injected, a partial inhibition of the initial velocity was observed. This experiment indi-

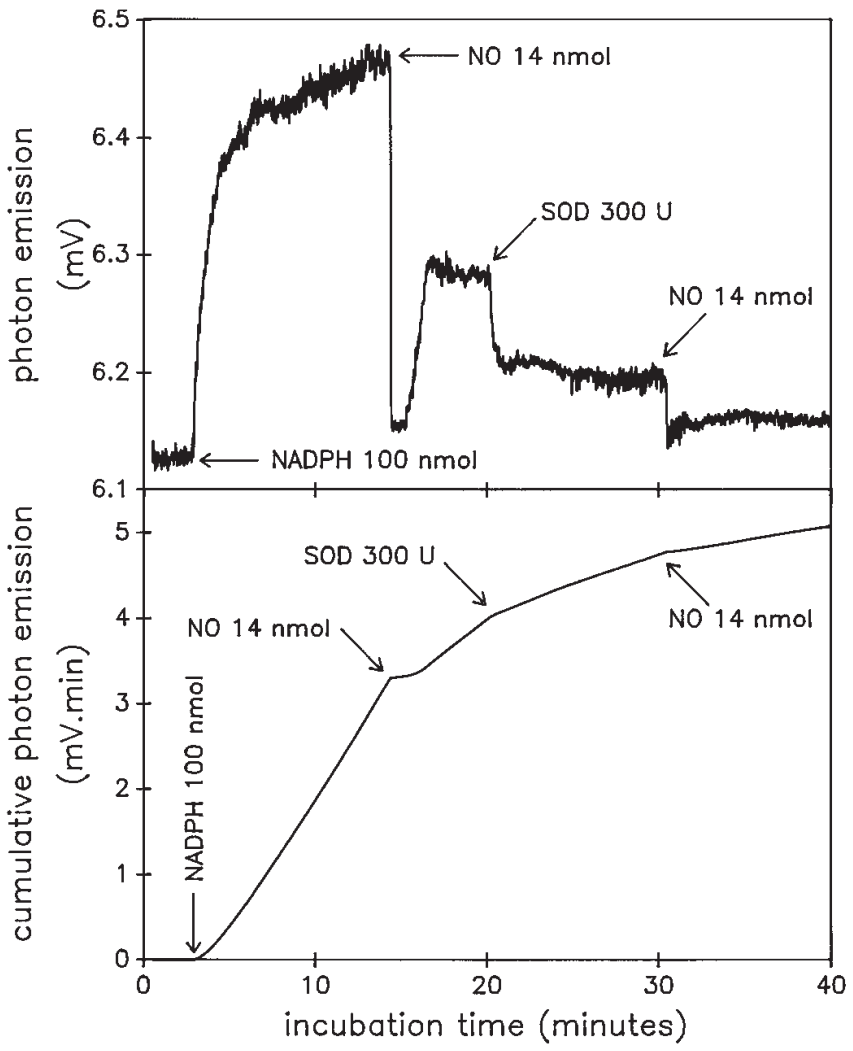

Figure 6. Inhibition of NADPH oxidase activity of rat aortic ring by direct NO injection. Upper panel: experimental recording of $\mathrm{CL}$ production induced and modulated by successive injections of 100 nmol NADPH, $14 \mathrm{nmol} \mathrm{NO}$ and $300 \mathrm{U}$ SOD in a $1 \mathrm{~mL}$ cuvette containing one rat aortic ring preincubated with $250 \mathrm{nmol} \mathrm{Luc}{ }^{2+}$ at $37^{\circ} \mathrm{C}$ Lower panel: initial velocities of photon production are calculated as indicated above. Units are those used in figure 5.

cated that added NO is able to react both with the extracellular and intracellular productions of $\mathrm{O}_{2}^{-}$and in contrast, injected SOD is only able to react with extracellular production of $\mathrm{O}_{2}^{-}$. These effects obtained with a direct injection of NO were similar to those observed by Tarpey et al. [10] on vessel $\mathrm{CL}$ using coelenterazine as an $\mathrm{O}_{2}^{-}$probe.

\section{Calibration of the $m V$ unit of $C L$ initial velocity}

In order to connect $\mathrm{mV}$ unit of $\mathrm{CL}$ initial velocity to a biochemical parameter, a calibration curve using the combination xanthine oxidase + xanthine as a source of $\mathrm{O}_{2}^{-}$was plotted comparing $\mathrm{CL}$ initial velocity and cytochrome $\mathrm{C}$ reduction initial velocity (Fig. 7). If the stoichiometry of the reaction is respected (one molecule of $\mathrm{O}_{2}^{-}$reduced one molecule of cytochrome $\mathrm{C}$ ), $1 \mathrm{mV}$ of $\mathrm{CL}$ initial velocity is equivalent to $800-900$ pmol reduced cytochrome $\mathrm{C}$ per $\mathrm{mL}$ and per min. The use of initial velocities (CL versus cytochrome $\mathrm{C}$ reduction) for the comparative plot is a crucial prerequisite for the relevance of this experimental comparative factor. 


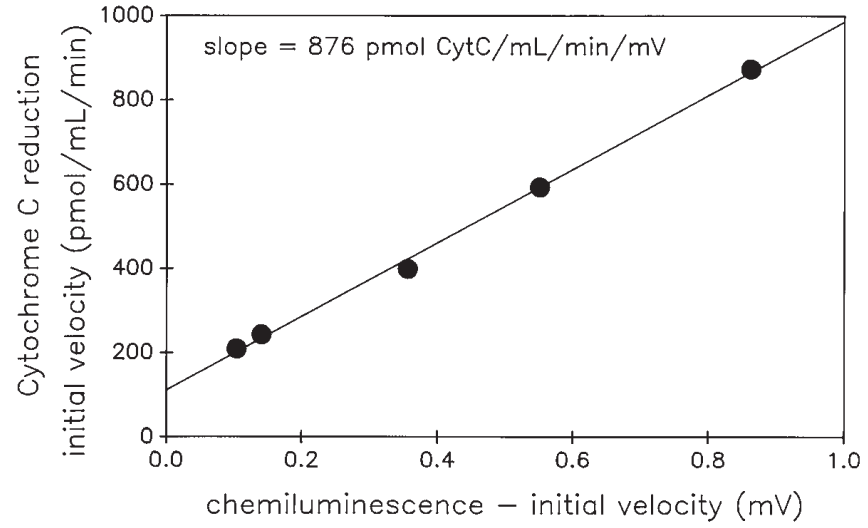

Figure 7. Calibration curve of $\mathrm{CL}$ initital velocity using cytochrome C reduction. Using xanthine oxidase $(100 \mu \mathrm{U} / \mathrm{mL}$ to $2 \mathrm{mU} / \mathrm{mL})+$ xanthine $(30 \mu \mathrm{M})$ as a source of $\mathrm{O}_{2}^{-}$, both cytochrome $\mathrm{C}(30 \mu \mathrm{M})$ reduction and lucigenin $(250 \mu \mathrm{M})$ were measured. The initial velocities obtained for each technique and identical xanthine oxidase concentrations are plotted. The slope value indicates that $1 \mathrm{mV}$ of $\mathrm{CL}$ initial velocity is equivalent to $800-900 \mathrm{pmol} \mathrm{O}_{2}^{-}$produced per min and per $\mathrm{mL}$.

\section{Characterisation of the Michaelis-Menten behaviour of rat aortic NADPH oxidase}

Rat aortic rings were exposed to various concentrations of NADPH alone or in the presence of SOD. The initial velocities were calculated as indicated above and plotted for each substrate concentration (Fig. 8). The Michaelis-Menten behaviour of NADPH-dependent CL production is characterised by the linearity of the Lineweaver-Burk double reciprocal plot. SOD acted as a non-competitive inhibitor. The $\mathrm{Km}$ and Vmax values for NADPH oxidase in rat aorta were $237 \pm 3 \mu \mathrm{M}$ and $0.716 \mathrm{mV}$ (CL fraction inhibited by SOD injection), respectively.

\section{Influence of physiopathological status on Michaelis-Menten behaviour of NADPH oxidase from mouse thoracic aortae}

As indicated in figure 8 for rat aortic rings, SOD acted also as a non competitive inhibitor (data not shown) on aortae from normal $(\mathrm{C} 57 \mathrm{bl})$ or hypercholestolemic mice (ApoE $\mathrm{KO})$. The comparison of ApoE $\mathrm{KO}$ mice versus normal C57 black mice is illustrated in figure 9 using the direct plot and the double reciprocal plot of the fraction of initial velocity inhibited by SOD injection in function of NADPH concentration. Control and hypercholesterolemic mice exhibited equivalent $\mathrm{Km}$ in the range 260-380 $\mu \mathrm{M}$. In contrast, Vmax increased by $77 \%$ in ApoE $\mathrm{KO}$ animals in comparison to C57 black mice. These data indicated that the deletion of the gene coding for an efficient cholesterol reverse transport did not modify the affinity of vascular NADPH oxidase for its substrate but increased the catalytic activity. Two simple hypothesis can be formulated to explain this observation: (1) an increased amount of the total NADPH oxidase
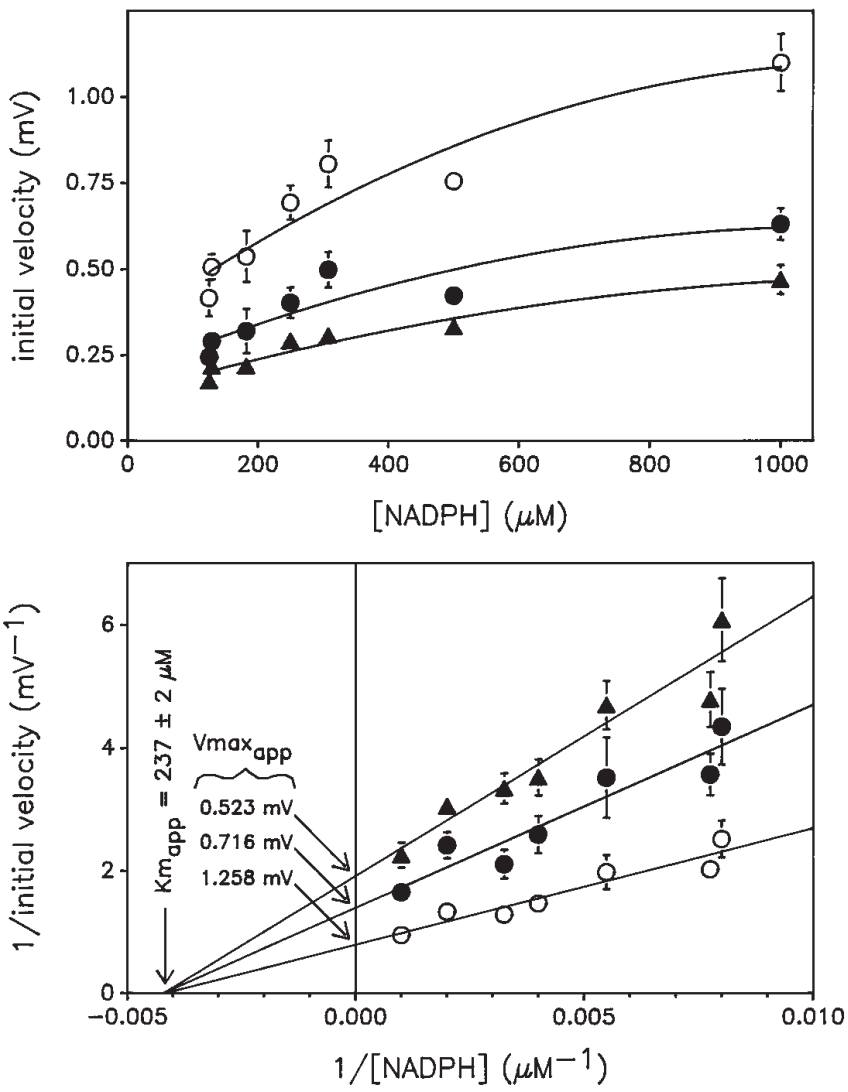

Figure 8. Michaelian parameters of NADPH oxidase activity of rat aortic ring. Experimental conditions are described in figure 5. Initial velocities are measured for a range of NADPH concentrations from $125 \mu \mathrm{M}$ to $1 \mathrm{mM}$. Open circles: initial velocity after NADPH injection. Solid triangles: initial velocity after SOD injection. Solid circles: fraction of initial velocity inhibited by SOD injection. Upper panel: plot of initial velocities in function of NADPH concentration. Lower panel: double reciprocal plot. SOD acted as a noncompetitive inhibitor. Apparent $\mathrm{Km}$ and $\mathrm{Vmax}$ values are indicated. Initial velocities are expressed as mean \pm sem (4-8 aortic rings).

enzyme in ApoE KO mice versus control mice and/or (2) a more efficient endogenous SOD activity in control mice versus ApoE KO mice.

If this data treatment method allows characterising Michaelian parameters of $\mathrm{NAD}(\mathrm{P}) \mathrm{H}$ oxidase from vascular preparation, this procedure does not give information about the intrinsic production of $\mathrm{O}_{2}^{-}$This method has also been used for the characterisation of $\mathrm{NAD}(\mathrm{P}) \mathrm{H}$ oxidase from intact porcine aortic endothelial cells (data not shown). Moreover, this procedure can help to describe the abnormal $\mathrm{NAD}(\mathrm{P}) \mathrm{H}$ oxidase activity of physiopathological cultured vascular cells such as regenerated endothelial cells after angioplasty [19] and smooth muscle cells exposed to shear stress [15].

For the illustration of the data treatment, experimental examples used $250 \mu \mathrm{M} \mathrm{Luc}{ }^{2+}$. Fridovich group [11,20] and 

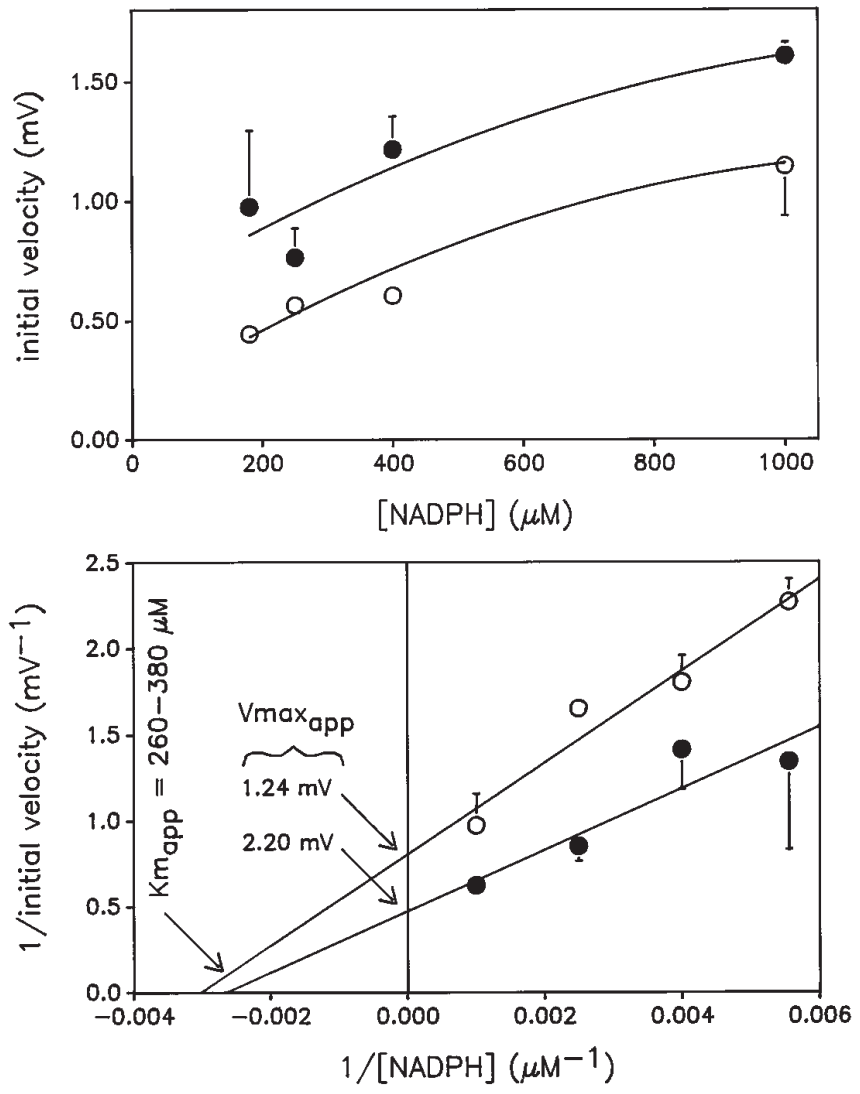

Figure 9. Comparison of thoracic aorta NADPH oxidase activity between hypercholesterolemic mice (ApoE KO) and control mice (C57bl). Experimental conditions are similar to those used in figure 8. One aorta (including arch) is incubated in $1 \mathrm{~mL}$ cuvette. Upper panel: direct plot for the fraction of initial velocity inhibited by SOD injection in function of NADPH concentration. Lower panel: double reciprocal plot for the fraction of initial velocity inhibited by SOD injection. Open circles: C57bl mice. Solid Circles: ApoE KO mice. Apparent Vmax values: $1.24 \mathrm{mV}$ for C57bl mice and $2.20 \mathrm{mV}$ for ApoE KO mice. Apparent $\mathrm{Km}$ values are in the range $260-380 \mu \mathrm{M}$. Initial velocities are expressed as mean \pm sem (3-4 animals).

others authors $[10,12]$ demonstrated these last years that at this level of concentration, Luc ${ }^{2+}$ can acted, under specific conditions, after initiation of photon production as an autogenerating source of $\mathrm{O}_{2}^{-}$. This inconvenient can be moderate for the experimental data presented in figure 9 indicating that Vmax of NADPH oxidase from pathological aortae were near $80 \%$ higher than Vmax of normal arteries. In this case, the absolute values of calculated Vmax include obviously artifactual $\mathrm{O}_{2}^{-}$production but the relative ratio of the comparison between the 2 vessel groups remains valid. In complex biological structures such as vessels or vascular cells, the global flow of $\mathrm{O}_{2}^{-}$production results from the difference between its local production and its consumption. In absence of $\mathrm{NO}$, the main pathways involved in $\mathrm{O}_{2}^{-}$ catabolism are SOD activities. This point is important because Fridovich group $[11,20]$ claimed that if $\mathrm{Luc}^{2+} \mathrm{CL}$ cannot be used validly for direct evaluation of vascular $\mathrm{O}_{2}^{-}$ production, it can be considered as a correct method for evaluation of SOD activity. The comparison between hypercholestolemic and normal mice illustrates this case as shown in figure 9. The limitations linked to Luc ${ }^{2+}$ utilisation are not important in regard to the topic of the present paper about CL data treatment because initial velocities of photon production by rat aortic rings have also been calculated (data not shown) from recordings utilising coelenterazine. This molecule is a CL probe without artifactual $\mathrm{O}_{2}^{-}$generation [10].

\section{Conclusions}

A method allowing a direct and easy measurement of endogenous production of vascular $\mathrm{O}_{2}^{-}$remains to be invented. All the conventional methods used in the literature are, such as CL, indirect and induced specific artifacts. Cytochrome $\mathrm{C}$ is a nonspecific electron acceptor. The use of added SOD as a proof of cytochrome $\mathrm{C}$ reduction by $\mathrm{O}_{2}^{-}$ needs the concomitant addition of catalase because $\mathrm{H}_{2} \mathrm{O}_{2}$ formed by $\mathrm{O}_{2}^{-}$dismutation oxidizes cytochrome $\mathrm{C}$ previously reduced by $\mathrm{O}_{2}^{-}$[21]. Electron spin resonance detection of $\mathrm{O}_{2}^{-}$needs very high concentrations of spin-trap (to 100-200 mM range) for the generation of detectable spinadduct [22]. The spin-trap is obviously a molecule with enhanced redox properties. What is the direct effect of 100$200 \mathrm{mM}$ spin-trap on redox status of treated vascular preparation? Fluoreogenic oxidation of hydroethidine to ethidium by $\mathrm{O}_{2}^{-}$is also a source of artifacts. This method uses the oxidative properties of $\mathrm{O}_{2}^{-}$linked to the transient generation of very labile $\mathrm{O}_{2}^{2-}$, instantaneously transformed into $\mathrm{H}_{2} \mathrm{O}_{2}$ in the presence of protons. Hydrogen peroxide is also able to oxidize hydroethidine but at a lower rate than $\mathrm{O}_{2}^{-}$. Benov et al. [23] indicated that ethidium oxidation can be considered only as a non-quantitative index of $\mathrm{O}_{2}^{-}$production for brief experimentation periods (2-15 minute range). In contrast, in the literature, ethidium detection of vascular $\mathrm{O}_{2}^{-}$is always described with incubation time higher than 45-60 minutes [19]. These remarks emphasise the real need of a direct detection of vascular $\mathrm{O}_{2}^{-}$Electrochemical procedures with specific electrodes would be considered as a promised technique for in situ detection of endogenous $\mathrm{O}_{2}^{-}$in vascular preparations but this technique, both delicate and timespending, allowed only a very little number of measurements per day [24].

Meanwhile, even if the endogenous production of vascular $\mathrm{O}_{2}^{-}$is not estimated, the use of integral calculus gives specific and precise information concerning the MichaelisMenten parameters of vascular $\mathrm{O}_{2}^{-}$production catalysed by $\mathrm{NAD}(\mathrm{P}) \mathrm{H}$ oxidase. This simple and computerised treatment of CL data could help to explain the subtle mechanisms of endothelial dysfunction induced by physiopathological conditions. 


\section{Acknowledgements}

The authors thank Dr. Saik Urien (Laboratoire de Pharmacologie, Faculté de Médecine, Créteil, France) for the editing of the CL data treatment software.

\section{References}

1. Gryglewsky, R. J.; Palmer, R. M. J.; Moncada, S. Nature Lond. 1986, 320, 454-456.

2. Ronson, R. R.; Nakamura, M.; Vinten-Johansen J. Cardiovasc. Res. 1999, 44, 47-59.

3. Griendling, K. K.; Minieri, C. A.; Ollerenshaw, J.D.; Alexander, R.W. Circ. Res. 1994, 74, 1141-1148.

4. Pagano, P.J.; Ito, Y.; Tornheim, K.; Gallop, P. M.; Tauber, A. I.; Cohen, R. A. Am. J. Physiol. 1995, 268, H2274-H2280.

5. Pagano, P. J.; Chanock, S. J.; Siwik, D. A.; Colucci, W. S.; Clark, J. K. Hypertension 1998, 32, 331-337.

6. Stroes, E. S. G.; van Faassen, E. E.; van Londen, G. J.; Rabelink, T. J. J. Cardiovasc. Pharmacol. 1998, 32, S14-S21.

7. Wever, R. M. F.; Lüscher, T. F.; Cosentino, F.; Rabelink, T.J. Circulation, 1998, 97, 108-112.

8. McCord, J. M.; Fridovich, I. J. Biol.Chem. 1969, 244, 60496055.

9. Shimomura, O.; Wu, C.; Murai, A.; Nakamura, H. A. Anal. Biochem. 1998, 258, 230-235.

10. Tarpey, M. M.; Roger, White C.; Suarez, E.; Richardson, G.; Radi, R.; Freeman, B. A. Circ. Res. 1999, 84, 1203-1211.
11. Liochev, S.I.; Fridovich, I. Arch. Biochem. Biophys. 1997, 337, 115-120.

12. Li, Y.; Zhu, H.; Kuppusamy, P.; Roubaudt, V.; Zweier, J.L.; Trush, M.A. J. Biol. Chem. 1998, 273, 2015-2023.

13. Michaelis, L.; Menten, M. L. Biochem. Z. 1913, 49, 333-340.

14. Villeneuve, N.; Bedioui, F.: Voituriez, K.; Avaro, S.; Vilaine, J.-P. J. Pharmacol. Toxicol. Meth. 1999, 40, 95-100.

15. Chiu, J. J.; Wung, B. S.; Shyy, J. Y. J.; Hsieh, H. J.; Wang, D. L. Arterioscler. Thromb. Vasc. Biol. 1997, 17, 3570-3577.

16. Nunes, G. L.; Robinson, K.; Kalynych, A.; King, III S. B.; Sgoutas, D. S.; Berck, B. C. Circulation, 1997, 96, 3593-3601.

17. Wang, H. D.; Hope, S.; Du, Y.; Quinn, M. T.; Cayatte, A.; Pagano, P. J.; Cohen, R. A. Hypertension, 1999, 33, 12251232.

18. Ohara, Y.; Peterson, T. E.; Harrison, D.G. J. Clin. Invest. 1993, 91, 2546-2551.

19. Fournet-Bourguignon, M. P.; Castedo-Delrieu, M.; Bidouard, J.P.; Leonce, S.; Saboureau, D.; Delescluse, I.; Vilaine, J.P.; Vanhoutte, P.M. Circ. Res. 2000, 86, 854-861.

20. Liochev, S.I.; Fridovich, I. Proc. Natl. Acad. Sci. USA, 1997, 94, 2891-2896.

21. Vandewalle, P. L.; Peterson, N. O. Febs Letters 1987, 210, 195-198.

22. Souchard, J. P.; Barbacanne, M. A.; Margeat, E.; Maret, A.; Nepveu, F.; Arnal, J. F. Free Rad. Res. 1998, 29, 441-449.

23. Benov, L.; Szteinberg, L.; Fridovich, I. Free Radic. Biol. Med. 1998, 25, 826-831.

24. Privat, C. Ph. D. Thesis, University of Paris VI, October 1999.

25. Beckman, J.S.; Chen, J.; Ischiropoulos H.; Crow J.P. Meth. Enzymol. 1994, 233, 229-240. 